\title{
Herzlich Willkommen in Wiesbaden!
}

D er 6. Deutsche Allergiekongress des Ärzteverbands Deutscher Allergologen (ÄDA), der Deutschen Gesellschaft für Allergologie und klinische Immunologie (DGAKI) und der Gesellschaft für Pädiatrische Allergologie und Umweltmedizin (GPA) findet vom 8. bis 10. September $2011 \mathrm{im}$ Kurhaus Wiesbaden statt. Die drei Fachgesellschaften bieten mit dieser Tagung ein interdisziplinäres Forum für die faszinierenden Fortschritte im sicherlich spannendsten Teilbereich der klinischen Medizin. Eine besondere Würdigung im Programm erfährt die spezifische Immuntherapie, die 1911 von Leonhard Noon erstmals beschrieben wurde und in diesem Jahr genau 100 Jahre jung wird. Neben der Vorstellung innovativer Methoden der Immuntherapie stehen Themen wie chronische Entzündungen im Bereich des Respirationstrakts, der Haut, des Gastrointestinaltrakts und Besonderheiten von Allergien bei Kindern sowie Tipps und Tricks in der Allergiediagnostik im Mittelpunkt.

Besonderes Augenmerk wird auf die praktische Fortbildung gelegt. Die von Prof. Dr. Thomas Werfel 2010 in Han-

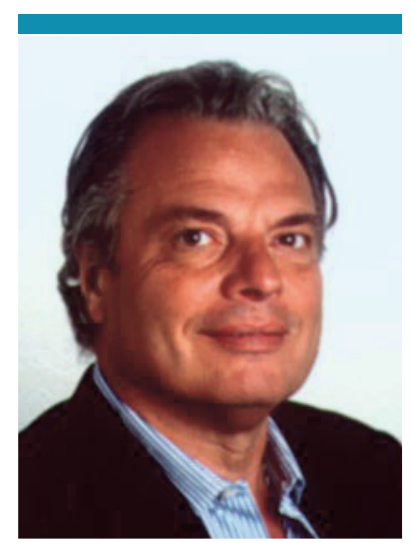

Prof. Dr. Wolfgang W. Schlenter, HNO-Abteilung, Sankt-MarienKrankenhaus, Frankfurt/Main

Vortrag oder eine Posterpräsentation ein!

Die historischen Ballräume des Kurhauses wie die gesamte Anlage mit Staatstheater und denkmalgeschütztem Parkensemble werden in die Veranstaltung mit einbezogen: sicherlich ein ideales Ambiente zum Wohlfühlen und zum kollegialen Austausch in angenehmer Atmosphäre. Nicht zuletzt beginnt im September im Rheingau die Weinlese - auch das ein Argument für ein Treffen zu dieser Zeit in Wiesbaden.

Im Namen des Organisationskomi-

nover etablierten Programmstrukturen mit Pro-und-Kontra-Sitzungen - zwei „Streithähne“, ein Schiedsrichter -, interaktiven Seminaren in Kleingruppen und spannenden Kasuistiken für Sherlock Holmes sorgen auch in Wiesbaden für Abwechslung. Werden Sie selbst Teil des internationalen Referententeams: Reichen Sie einen Abstract für einen<smiles>C=CC(C)CC</smiles>

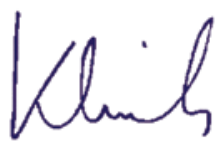

Prof. Dr. Ludger Klimek tees und der beteiligten Gesellschaften laden wir Sie herzlich zum 6. Deutschen Allergiekongress nach Wiesbaden ein. Wir freuen uns auf eine intensive Fortbildung und anregende Diskussionen gemeinsam mit Ihnen! Weitere Informationen zur Anmeldung und zur Abstracteinreichung finden Sie unten.

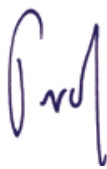

Prof. Dr. Wolfgang Schlenter

\section{Deutscher Allergiekongress auf einen Blick}

\section{Veranstalter}

Ärzteverband Deutscher Allergologen (ÄDA)

Deutsche Gesellschaft für Allergologie und klinische Immunologie (DGAKI)

Gesellschaft für Pädiatrische Allergologie und Umweltmedizin (GPA)

vertreten durch AKM Allergiekongress und Marketing $\mathrm{GmbH}$, Solingen

Kongresstermin

8.-10. September 2011

\section{Kongressort}

Kurhaus Wiesbaden Kurhausplatz 1, 65189 Wiesbaden

\section{Wissenschaftliche Leitung}

Prof. Dr. Ludger Klimek, Wiesbaden

Prof. Dr. Wolfgang Schlenter, Frankfurt/Main

\section{Kongresssekretär}

PD Dr. Oliver Pfaar, Allergiezentrum

Wiesbaden, An den Quellen 10, 65183 Wiesbaden

Tel.: (o6 11) 50595257

Fax: (06 11) 50595295

E-Mail: wiesbaden2011@allergiekongress.de

Kongressorganisation und Auskunft wikonect $\mathrm{GmbH}$, Hagenauer Straße 53, 65203 Wiesbaden

Tel.: (06 11) 2048 o9-o

Fax: (06 11) 2048 o9-10

E-Mail:info@wikonect.de

\section{Anmeldung und Information}

Anmeldung mit nebenstehendem Formular oder unter www.allergiekongress.de

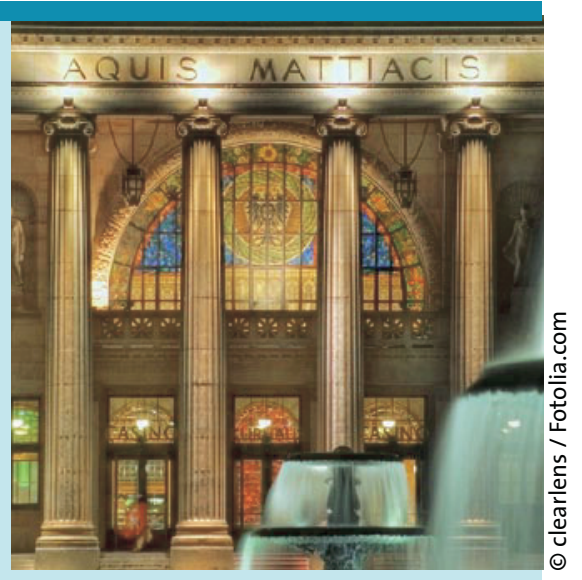

Abstracteinreichung

Wissenschaftliche Beiträge können von 1. März bis 31. Mai online eingereicht werden unter http://abstracts.urban-vogel.de. 